\title{
Dynamical Elliptical Diagnostics of the Antarctic Polar Vortex
}

\author{
M. MESTER AND J. G. ESLER \\ University College London, London, United Kingdom
}

(Manuscript received 27 August 2019, in final form 10 December 2019)

\begin{abstract}
Elliptical diagnostics provide dynamical and climatological information about the behavior of the Arctic and Antarctic stratospheric polar vortices. Here Kida's model, describing the evolution of a uniform vortex in a linear, but possibly unsteady, background flow, is used to interpret the observed evolution of the Antarctic vortex in late winter during 1999-2018. Kida's model has oscillatory solutions that can undergo an amplitude bifurcation, which serves as a simple model for the onset of vortex-splitting stratospheric sudden warmings (SSWs). A data assimilation method is used to find solutions of Kida's equations consistent with the observations. A phase-plane analysis reveals large interannual variability in the amplitude of oscillations of the vortex. In 2002, the year of the only observed vortex-splitting Antarctic SSW, the system is found to cross a separatrix in phase space, associated with the SSW amplitude bifurcation, in late September. An output of the data assimilation is the linear background flow experienced by the vortex. The rotational component of this linear flow is consistent with the vortex being embedded in an anticyclonic background. The time-mean strain flow is weak but has a clear orientation, consistent with the presence of stationary forcing due to planetary-scale topography and land-sea contrast. The time-varying strain flow is comparatively large in magnitude, illustrating the relative importance of the planetary-scale component of the turbulent dynamics occurring at tropopause level. Unlike in the Northern Hemisphere, therefore, the direction of future Antarctic vortex splits will not necessarily align with the direction of the 2002 split.
\end{abstract}

\section{Introduction}

Elliptical diagnostics were introduced as a method to determine compact and fundamental information about the state of the Arctic and Antarctic stratospheric polar vortices by Waugh (1997). The basic idea is to take an available chemical [e.g., nitrous oxide $\left(\mathrm{N}_{2} \mathrm{O}\right)$ ] or dynamical (e.g., isentropic potential vorticity) tracer field on a given level in the stratosphere, and make a "best fit" of a piecewise constant function with an elliptical boundary to this field, with the aim of capturing the vortex location, shape, and orientation. The best fit ellipse is calculated using the integral moments of the field in question, and then choosing the ellipse to match. The resulting time series of the vortex centroid, area, aspect ratio, and orientation provide a detailed picture of the climatology, interannual variability, seasonal cycle, and vertical structure of the vortices in each hemisphere [see, in particular, Waugh and Randel (1999)]. Subsequent authors (e.g.,

Corresponding author: M. Mester, marton.mester.16@ucl.ac.uk
Matthewman et al. 2009; Hannachi et al. 2011) have used these diagnostics to study the vortex evolution in the lead-up and aftermath of stratospheric sudden warmings (SSWs) and to investigate statistical extremes of the vortices (Mitchell et al. 2011). The vortex moment diagnostics underpinning elliptical diagnostics have also proved useful in quantitatively evaluating the representation of the polar vortices in different chemistry-climate models (Mitchell et al. 2012), and even in assessing the nature of polar vortices on Mars (Mitchell et al. 2015).

All of the studies above are concerned with the observed statistics of the stratospheric vortices. Here, the aim is to go further by using the elliptical diagnostics to understand the dynamical evolution of the vortex, by making use of a simple model. The model in question is that of Kida (1981), which describes the evolution of a two-dimensional vortex of constant vorticity in a linear, but possibly unsteady, background flow. The original suggestion for using Kida's model in this way is in fact due to Waugh (1997). Of course, Kida's model cannot be expected to capture every aspect of the dynamics of a 
three-dimensional vortex, in a spherical geometry, embedded in a turbulent planetary atmosphere; however, it may nevertheless describe much of the essential dynamics. Evidence of this is provided by Polvani et al. (1990), who found that Kida's model provides an excellent quantitative explanation of the observed evolution of Neptune's Great Dark Spot, as observed during the Voyager 2 flypast.

If Kida's model is to be used to interpret observations, the key question is exactly how the linear flow should be chosen to fit the model to the data. In the case of Neptune's Great Dark Spot (at least during the Voyager 2 flypast), the vortex evolution is sufficiently coherent and predictable that a time-independent linear flow (in fact a simple shear flow) is sufficient to give a good fit to the data. The stratospheric polar vortices on Earth, however, are considerably less predictable. The Arctic vortex in particular exhibits significant variability in its vertical structure, and consequently the two-dimensional Kida model cannot be expected to capture key aspects of its dynamics. By contrast, disturbances of the Antarctic vortex typically remain coherent in the vertical, particularly during late winter (August-September). The current study is therefore restricted to the August-September behavior of the Antarctic vortex over the 20-yr period 1999-2018, with a particular focus on 2002, the year of the only observed vortex-splitting Antarctic SSW [see, e.g., J. Atmos. Sci. special issue 62 (3), 2005]. It is well established (e.g., Scaife et al. 2005; Esler and Mester 2019, EM19 hereafter) that in at least some years (e.g., 2012, 2016) the Antarctic vortex has been observed to undergo Kida-like oscillations, but in other years (e.g., 2014, 2015) these appear to be largely absent.

The aim of this work is to use a variational data assimilation technique to combine the observed elliptical diagnostics with the Kida model, and to apply the technique to the Antarctic late winter over the periods detailed above. In doing so, the slowly evolving background linear flow that is most consistent with model and data is determined. The background linear flow can be expected to have several components:

- A slowly evolving rotational component, which is anticyclonic, and relatively large in magnitude. This anticyclonic rotation is due to the relative vorticity in the stratospheric surf-zone surrounding the vortex having the opposite sign to the Coriolis parameter.

- A slowly evolving strain flow component that is due to stationary planetary waves forced by surface topography, land-sea contrast, etc.

- A more rapidly evolving strain and rotational flow component that is due to "tropospheric macroturbulence," that is, planetary-scale waves generated by the interaction of baroclinic eddies at tropopause level (see, e.g., Scinocca and Haynes 1998).

One specific goal is to assess the interannual variability of the background linear flow components above, with the aim of improving understanding of the causes of vortex-splitting SSWs.

In section 2 Kida's model is described and a Hamiltonian formalism is introduced, allowing a phase-portrait analysis of the observed vortex evolution, and clarifying the dynamical criterion used to identify the onset of a vortex-splitting SSW. The reanalysis dataset and elliptical diagnostics used to quantify the observed vortex behavior are then described. In section 3, the observed elliptical diagnostics are analyzed using the resulting phase-portrait method. In section 4 the variational data assimilation method is introduced and tested, and in section 5 the main results from the assimilation method are presented, and new perspectives on the interannual variability of the vortex and background flow emerge. Finally, in section 6, conclusions are drawn.

\section{Dynamical model, data, and methodology}

\section{a. A symmetric, nonsingular Hamiltonian form for Kida's model}

Kida's equations (Kida 1981; Dritschel 1990) describe the motion in two dimensions of an elliptic vortex in a linear background flow. The equations describe the time evolution of the aspect ratio $\lambda \geq 1$ and orientation $\theta$ of the vortex,

$$
\begin{aligned}
& \dot{\theta}=\Omega+\frac{\omega \lambda}{(\lambda+1)^{2}}-\frac{\lambda^{2}+1}{\lambda^{2}-1} \Gamma \sin (2 \theta-2 \phi), \\
& \dot{\lambda}=2 \lambda \Gamma \cos (2 \theta-2 \phi) .
\end{aligned}
$$

Here $\Omega$ and $\Gamma$, respectively, denote the magnitude of the rotational and strain components of the linear flow, $\phi$ is the angle of the strain axis, and $\omega$ is the vorticity. In the most general form of the equations each of $\Omega, \Gamma$, and $\phi$ are time dependent.

For the purposes of this work, it is helpful to consider a transformed version of Kida's equations. First, it is helpful to define a strain-rotation vector $\Gamma \equiv\left(\Gamma_{1}, \Gamma_{2}\right.$, $\left.\Gamma_{3}\right)^{\mathrm{T}}=[\Gamma \cos (2 \phi), \Gamma \sin (2 \phi), \Omega]^{\mathrm{T}}$. Notice that $\Gamma$ defines a general incompressible linear flow $\mathbf{u}=\mathcal{A} \cdot \mathbf{x}$ where the matrix $\mathcal{A}$ is given by

$$
\mathcal{A}=\left(\begin{array}{cc}
\Gamma_{1} & \Gamma_{2}-\Gamma_{3} \\
\Gamma_{2}+\Gamma_{3} & -\Gamma_{1}
\end{array}\right) .
$$

Next, following Melander et al. (1986), who use the same transformation in their derivation of a Hamiltonian 
moment model for two-dimensional vortex dynamics, introduce the ellipticity vector

$$
\mathbf{X} \equiv\left(\begin{array}{c}
X \\
Y
\end{array}\right)=\left(\lambda^{1 / 2}-\lambda^{-1 / 2}\right)\left(\begin{array}{c}
\cos 2 \theta \\
\sin 2 \theta
\end{array}\right) .
$$

It is useful to note the monotonic relationship between the magnitude $|\mathbf{X}|$ of the ellipticity vector and the aspect ratio $\lambda$,

$$
\lambda=\frac{1}{4}\left[|\mathbf{X}|+\left(|\mathbf{X}|^{2}+4\right)^{1 / 2}\right]^{2} .
$$

This relationship is plotted for convenience in Fig. 1. When there is no strain, it is well known that the vortex becomes unstable when $\lambda>3$ (Love 1893). In terms of the ellipticity vector, this criterion can be expressed as $|\mathbf{X}|>2 / \sqrt{3}$. When strain is present, the periodic orbits undergone by the vortex become more and more unstable as their amplitude increases, with the first morphological change in stability also occurring at $\lambda=3$ (Dritschel 1990).

It is a straightforward calculation to verify from (1) that $\mathbf{X}$ evolves according to Hamilton's equations:

$$
\dot{\mathbf{X}}=\nabla^{\perp} H,
$$

where $\nabla^{\perp} \equiv \mathbf{k} \times \nabla$ is the skew-gradient operator with respect to $\mathbf{X}$, and the Hamiltonian $H$ is

$$
\begin{aligned}
H(\mathbf{X})= & \left(|\mathbf{X}|^{2}+4\right)^{1 / 2} \boldsymbol{\Gamma} \cdot(\mathbf{k} \times \mathbf{X})+|\mathbf{X}|^{2} \boldsymbol{\Gamma} \cdot \mathbf{k} \\
& +\omega \log \left(\frac{|\mathbf{X}|^{2}+4}{4}\right) .
\end{aligned}
$$

The Hamiltonian $H$ is, up to a constant multiplicative factor and additive constant, equal to the conserved quantity of the motion found by Kida (1981) for the case of constant $\boldsymbol{\Gamma}$ and $\omega$ [also $H=-h$, as defined in (2) of EM19].

The Hamiltonian form (4) and (5) has a number of advantages over (1):

- In the standard form of the Kida equations [(1)], the circular vortex $(\lambda=1)$ is associated with a singularity because $\dot{\theta}$ becomes singular. In the transformed equation the circular vortex corresponds to $\mathbf{X}=0$ and the singularity is removed.

- The symmetric form of (4) and (5) allows for simple norms to be defined. For example, $\left|\mathbf{X}-\mathbf{X}_{\text {obs }}\right|$ can be used to measure the distance between a model solution and observed data. Also $|\boldsymbol{\Gamma}|$ is a physically meaningful measure of the magnitude of the linear flow $\mathbf{u}=\mathcal{A} \cdot \mathbf{x}$, because its energy in a circle of radius $R$ is proportional to $|\boldsymbol{\Gamma}|^{2} R^{2}$.

- The methods of Hamiltonian mechanics are available. For example, contour plots of the Hamiltonian allow

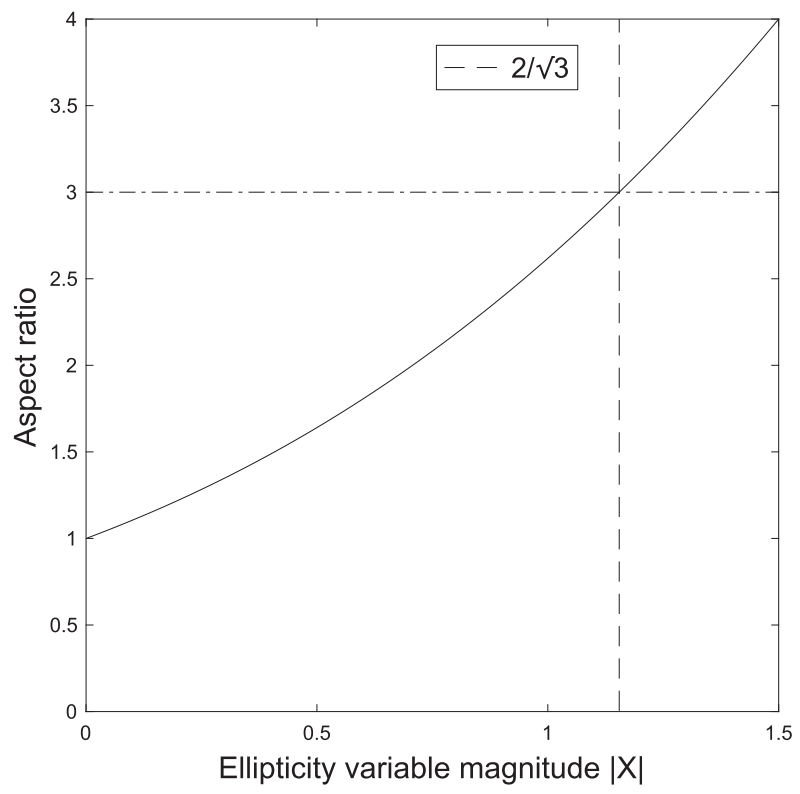

FIG. 1. Relation between aspect ratio and the magnitude of the ellipticity vector. Love's instability occurs for $\lambda \geq 3$ or equivalently $|\mathbf{X}| \geq 2 / \sqrt{3}$.

for a simple phase-portrait interpretation of the dynamics, including the bifurcation associated with an SSW, which will be exploited below.

The Hamiltonian phase-portrait representation, illustrated in Fig. 2, allows the qualitatively different vortex behaviors [see Dritschel (1990) and Matthewman and Esler (2011) for detailed discussion] to be easily differentiated. Note that, for ease of comparison with the Antarctic vortex, the portraits have been plotted using the convention that $\omega$ is negative (rather than positive as is typical). The top panels show the Hamiltonian for a flow defined by strain-rotation vector $\boldsymbol{\Gamma}$ that is typical of Southern Hemisphere wintertime conditions (see section 5 below). The key feature is the self-intersecting separatrix (bold line) defining the boundary between the highellipticity anticlockwise (ACW)-rotating regime, the low-ellipticity clockwise $(\mathrm{CW})$-rotating regime, and the intermediate oscillating (OSC) region where the vortex undergoes both a clockwise and anticlockwise rotation as it completes a full oscillation period. In Matthewman and Esler (2011) and EM19, the onset of an SSW is associated with crossing the separatrix from the $\mathrm{CW}$ to the OSC regimes, resulting in rapid growth in ellipticity, a reversal in the direction of vortex rotation, and in practice a split. It should be noted that crossing the separatrix at the point where it self-intersects may result in the vortex transitioning to the ACW regime instead of OSC.

Notice also that, within the $\mathrm{CW}$ regime there is a lowamplitude regime (NUT), where the orientation of the 

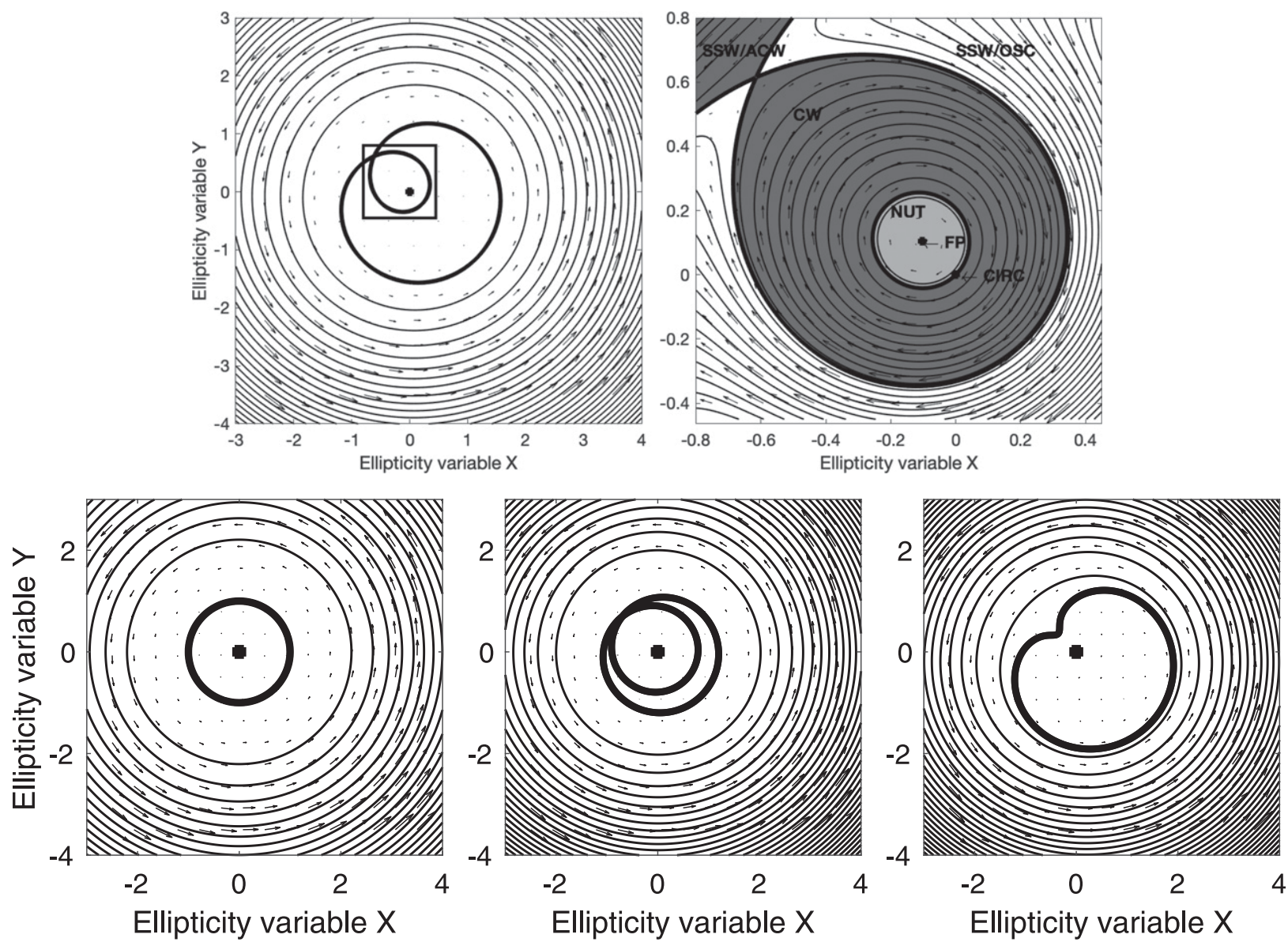

FIG. 2. Contours of the Hamiltonian for the representative case $\omega=-1, \Gamma_{1}=-0.005, \Gamma_{2}=-0.005$, and $\Gamma_{3}=0.2$. (top left) All the different possible evolutions, including ones with high aspect ratio. (top right) Zoom on the $\mathrm{CW}$ region that is associated with typical midwinter SH polar vortex evolution. (bottom left) In the absence of strain (i.e., $\boldsymbol{\Gamma}=0$ ), contours are circles and the vortex is either $\mathrm{CW}$ or ACW. (bottom center) In the presence of weak strain $\left(\Gamma_{1}=-0.0006, \Gamma_{2}=-0.0006\right)$, a self-intersecting separatrix emerges, with the CW regime located within the smaller loop and the OSC regime located between the two branches of the separatrix. (bottom right) For stronger strain $\left(\Gamma_{1}=-0.013, \Gamma_{2}=-0.013\right)$, the smaller loop and $\mathrm{CW}$ regime disappears.

vortex nutates around a fixed angle. The boundary between CW and NUT is given by the trajectory that passes through an initially circular vortex (CIRC), that is, through $\mathbf{X}=0$. The system also has a fixed point (FP) inside NUT.

The lower three panels show how the magnitude of the strain affects the Hamiltonian structure. In the absence of strain $\left(\Gamma_{1}=\Gamma_{2}=0\right)$ the phase lines are circular and the behavior is that of the well-known Kirchhoff vortex. When the strain is weak (bottom-center panel) the OSC regime within the separatrix contour occupies only a slender region of phase space. Finally, when the strain is strong, compared to that in the top panel, the $\mathrm{CW}$ regime (identified here with normal SH conditions) disappears completely.

\section{b. Dataset and vortex definition}

Our observational data is derived from the European Centre for Medium-Range Weather Forecasts (ECMWF) interim reanalysis (ERA-Interim) (Dee et al. 2011). The main datasets used are potential vorticity $q$ (PV hereafter) and relative vorticity $\zeta$ on eight isentropic $(375-850 \mathrm{~K})$ levels in the Southern Hemisphere with $0.7^{\circ} \times$ $0.7^{\circ}$ horizontal resolution. Climatologically, the Antarctic polar vortex attains its maximum area coverage during July-August, before decreasing slightly in September, and then more rapidly in October and November (see, e.g., Waugh and Randel 1999) before breaking up in a final warming around 1 December (Black and McDaniel 2007). Here, the focus is on the August-September period when the vortex is well established and supports significant planetary wave activity (July is more quiescent). It is during this period that Kida's model, which describes the motion of a vortex with constant area, is most relevant to the dynamics of the Antarctic vortex. The analysis focuses on the 20-yr period 1999-2018, including in particular 2002, when the only recorded Antarctic SSW occurred. 
The vortex is defined from the observed PV following Matthewman et al. (2009). Briefly, for each potential temperature level $\theta$, the average $\mathrm{PV} q_{b}(\theta, t)$ poleward of $45^{\circ} \mathrm{S}$ is calculated on this level for each available time $t$. The Antarctic vortex, while larger than its Arctic counterpart, nevertheless remains almost entirely within this region throughout the period of interest. The vortex boundary is then defined to be the PV surface where $q=q_{b}(\theta, t)$.

To apply the proposed Kida model to the polar vortex, it is necessary to calculate a representative value of $\omega$, which is the jump in relative vorticity across the vortex edge boundary. To do this, we exploit the vortex edge definition introduced above to calculate the average relative vorticity $\omega_{i}$ in the interior of the vortex, and the average relative vorticity $\omega_{o}$ between the latitude circle $45^{\circ} \mathrm{S}$ and the vortex boundary. Contour plots of relative vorticity (not shown) show more spatial variability than the PV shown in Fig. 4, but a piecewise constant fit remains a reasonable approximation. The jump in relative vorticity is then calculated as $\omega=\omega_{i}-\omega_{o}$. The evolution of $\omega(t)$ on the 600-K isentropic surface during the winters 2009-18 and 2002 is shown on Fig. 3. Each year, $\omega$ ranges between -7 and -4 day $^{-1}$ during the first half of August and between -8 and -5 day $^{-1}$ during late September. As a general trend, a slow decrease in $\omega$ during the August-September period can be clearly seen; however, such strengthening in the vorticity jump does not occur every year (e.g., 2002, 2010).

\section{c. Elliptical diagnostics}

Our PV-based construction of the equivalent ellipse follows the method described by Matthewman et al. (2009), which is a modification to the elliptical diagnostics framework applied by Waugh (1997). First, we use Lambert's azimuthal equal-area projection $(\lambda, \phi) \rightarrow(x, y)=R \sqrt{2(1-\sin \phi)}(\cos \lambda, \sin \lambda)$, where $\lambda$ is the longitude, $\phi$ is the negative of the latitude, and $R$ is the radius of Earth, to map the vortex boundary and the PV structure of the Southern Hemisphere to a plane from the spherical geometry. A slight advantage of this projection over the frequently used (e.g., in Waugh and Randel 1999) polar stereographic projection is that the latter underestimates the contribution of regions at lower latitudes. To calculate the diagnostics, the potential vorticity field is first modified according to

$$
\hat{q}(\mathbf{x}, \theta, t)=\left\{\begin{array}{cc}
q(\mathbf{x}, \theta, t), & q(\mathbf{x}, \theta, t)<q_{b}(\theta, t), \\
q_{b}(\theta, t), & q(\mathbf{x}, \theta, t) \geq q_{b}(\theta, t) ;
\end{array}\right.
$$

that is, low-latitude $\mathrm{PV}$ variations are neglected by setting the PV to be constant outside the vortex. Next, the $M_{k, l}(\theta, t)$ moment diagnostic is defined by the area integral

$$
M_{k, l}=\int_{\mathbb{R}^{2}}\left[\hat{q}(x, y)-q_{b}\right] x^{k} y^{l} d x d y .
$$

Note that the integrand vanishes outside of the vortex. Following Matthewman et al. (2009), the equivalent ellipse is defined to have a constant uniform interior PV $\left(q_{c}+q_{b}\right.$, where the value of $q_{c} \approx q_{b}$ enters only into the area calculation below), and is otherwise chosen to match the zeroth-, first-, and second-order moments of the observed vortex. First, the center of the equivalent ellipse is given by

$$
(\bar{x}, \bar{y})=\frac{1}{M_{0,0}}\left(M_{1,0}, M_{0,1}\right),
$$

and the relative vortex moments are defined to be

$$
J_{k, l}=\int_{\mathbb{R}^{2}}\left[\hat{q}(x, y)-q_{b}\right](x-\bar{x})^{k}(y-\bar{y})^{l} d x d y .
$$

Now, the orientation $\Theta$, and the aspect ratio $\Lambda$ of the equivalent ellipse are given by the formulas

$$
\begin{aligned}
& \Theta=\frac{1}{2} \tan ^{-1}\left(\frac{2 J_{1,1}}{J_{2,0}-J_{0,2}}\right), \\
& \Lambda=\left|\frac{J_{2,0}+J_{0,2}-\sqrt{4 J_{1,1}^{2}+\left(J_{2,0}-J_{0,2}\right)^{2}}}{J_{2,0}+J_{0,2}+\sqrt{4 J_{1,1}^{2}+\left(J_{2,0}-J_{0,2}\right)^{2}}}\right|^{1 / 2} .
\end{aligned}
$$

The obtained time series of $\Theta$ and $\Lambda$ are the basis of the interpretation using Kida's model. For illustrative purposes only, we may also define the area of the equivalent ellipse by $A=M_{0,0} / q_{c}$. Figure 4 shows the shape and position of the equivalent ellipse, constructed from the observed potential vorticity distribution on the $600-\mathrm{K}$ isentropic surface on 1 August 2002.

At each available time $t_{n},(2)$ is used to calculate the value of the ellipticity vector $\mathbf{X}_{n}$ from $(\Lambda, \Theta)$. Cubic interpolation is then used to generate an ellipticity vector $\mathbf{X}_{\text {obs }}(t)$ with a continuous first time derivative. While the vector $\mathbf{X}_{\mathrm{obs}}(t)$ is evidently a derived quantity from the reanalysis data, it serves as the observations in the data assimilation method to be described in section 4 below, and will be henceforth be referred to as the observed ellipticity vector.

\section{Observations of vortex evolution 1999-2018}

The observed ellipticity vector $\mathbf{X}_{\mathrm{obs}}(t)$ gives a new perspective on the evolution of the Antarctic vortex in the late winter. In particular the propagation of 


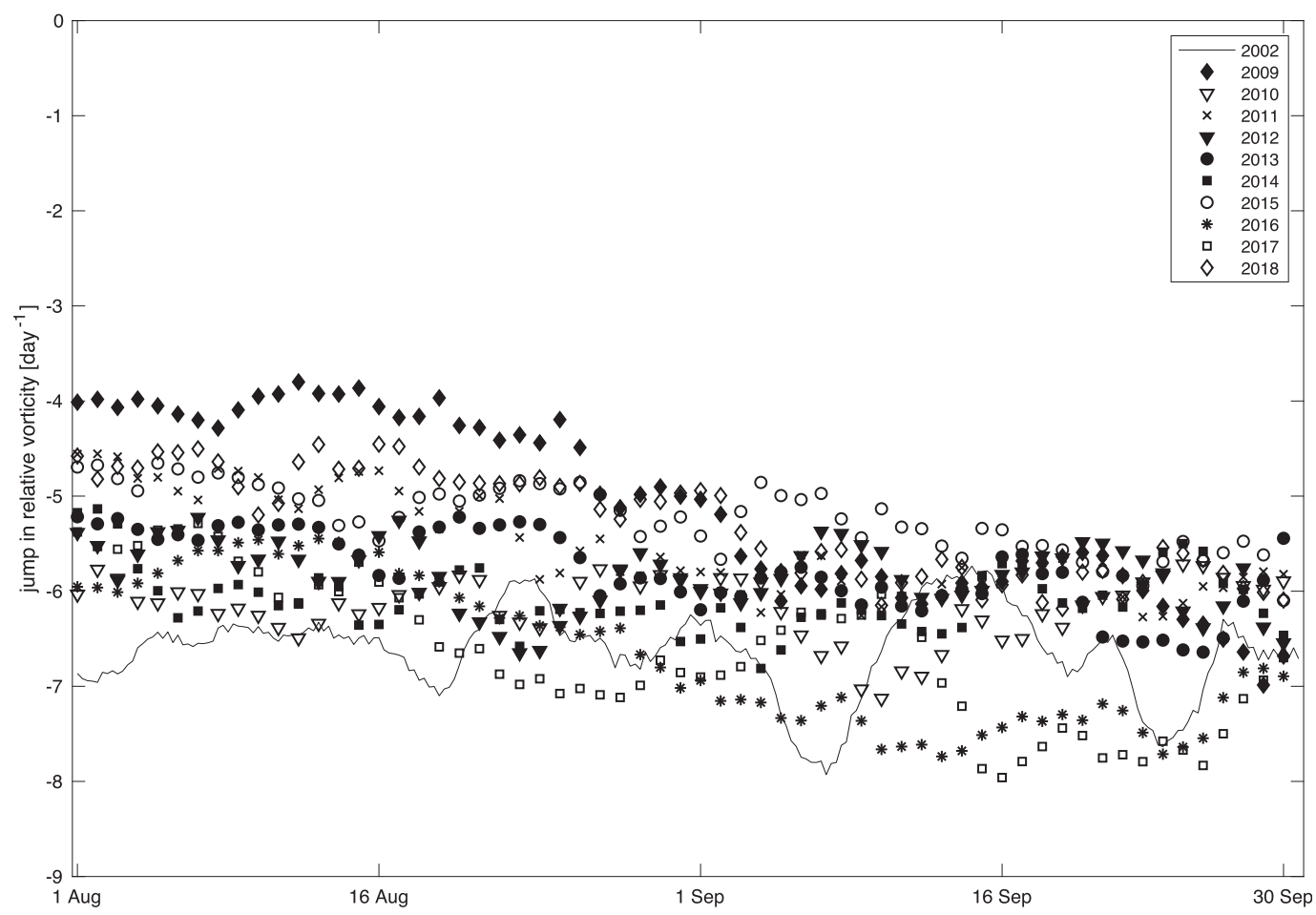

FIG. 3. The evolution of the vorticity jump on the 600-K isentropic surface during winters 2002 and 2009-18.

large-amplitude (wavenumber 2) Rossby waves on the vortex can be easily visualized, using the phase-space interpretation described above. Focusing first on the magnitude of the ellipticity vector, Fig. 5 shows the evolution of $\left|\mathbf{X}_{\text {obs }}(t)\right|$ during August-September 2009-18 and 2002 (curve with circles, other years 1999-2008 omitted for clarity) on the $600-\mathrm{K}$ isentropic level. The evolution of the ellipticity vector is found to be highly coherent in the vertical (plots of vertical structure not shown here), especially during periods when the vortex is relatively undisturbed. When the vortex is strongly disturbed, the ellipticity can increase significantly with height; however, the oscillations on different isentropic levels remain highly correlated. As a consequence, the results below are presented for the $600-\mathrm{K}$ surface, which is representative of the general behavior throughout the stratosphere. For each of the years shown $\left|\mathbf{X}_{\mathrm{obs}}(t)\right|$ has a relatively low magnitude in the first half of August, indicating a near-circular, undisturbed midwinter vortex. Subsequently, in several years significant oscillations develop. It is clear, at a glance, that the period immediately preceding the vortex split in 2002 is the only occasion when Love's criterion $\left|\mathbf{X}_{\text {obs }}\right|>2 / \sqrt{3}$ for vortex instability is met, which, while it is not an exact condition for an SSW onset for the real threedimensional vortex in spherical geometry, is evidently somewhat indicative of the onset of vortex instability.
(Note that the neglect of spherical geometric effects can be justified under the $f$-plane approximation.) Apart from 2002, the year in which $\left|\mathbf{X}_{\text {obs }}(t)\right|$ attains its highest value $(\approx 0.9)$ is 2017 (curve labeled with crosses). The climatological value of $\left|\mathbf{X}_{\text {obs }}\right|$ (thick black curve)

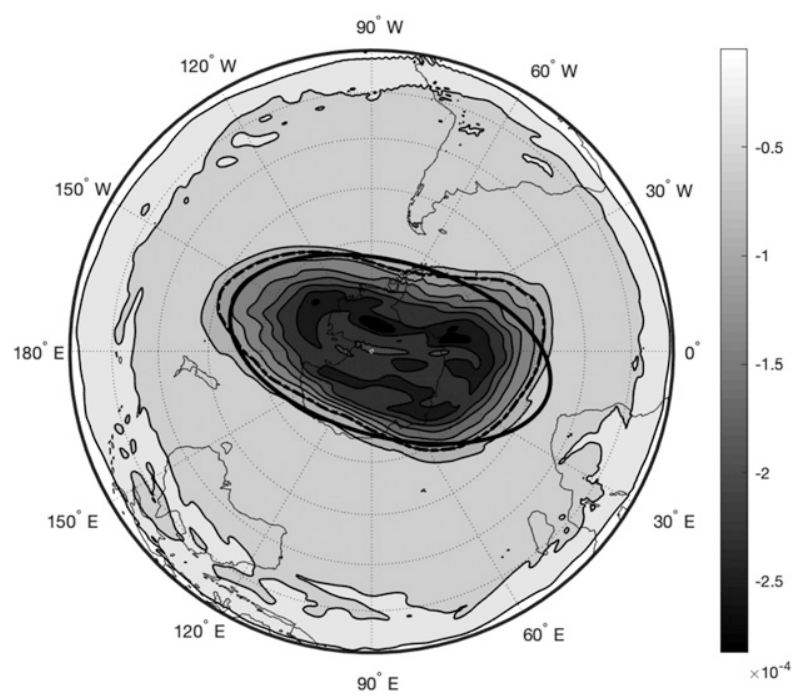

FIG. 4. PV contours $\left(\mathrm{km}^{2} \mathrm{~kg}^{-1} \mathrm{~s}^{-1}\right)$, vortex boundary (dashed), and equivalent ellipse (bold) on the 600-K isentropic surface on 1 Aug 2002. 


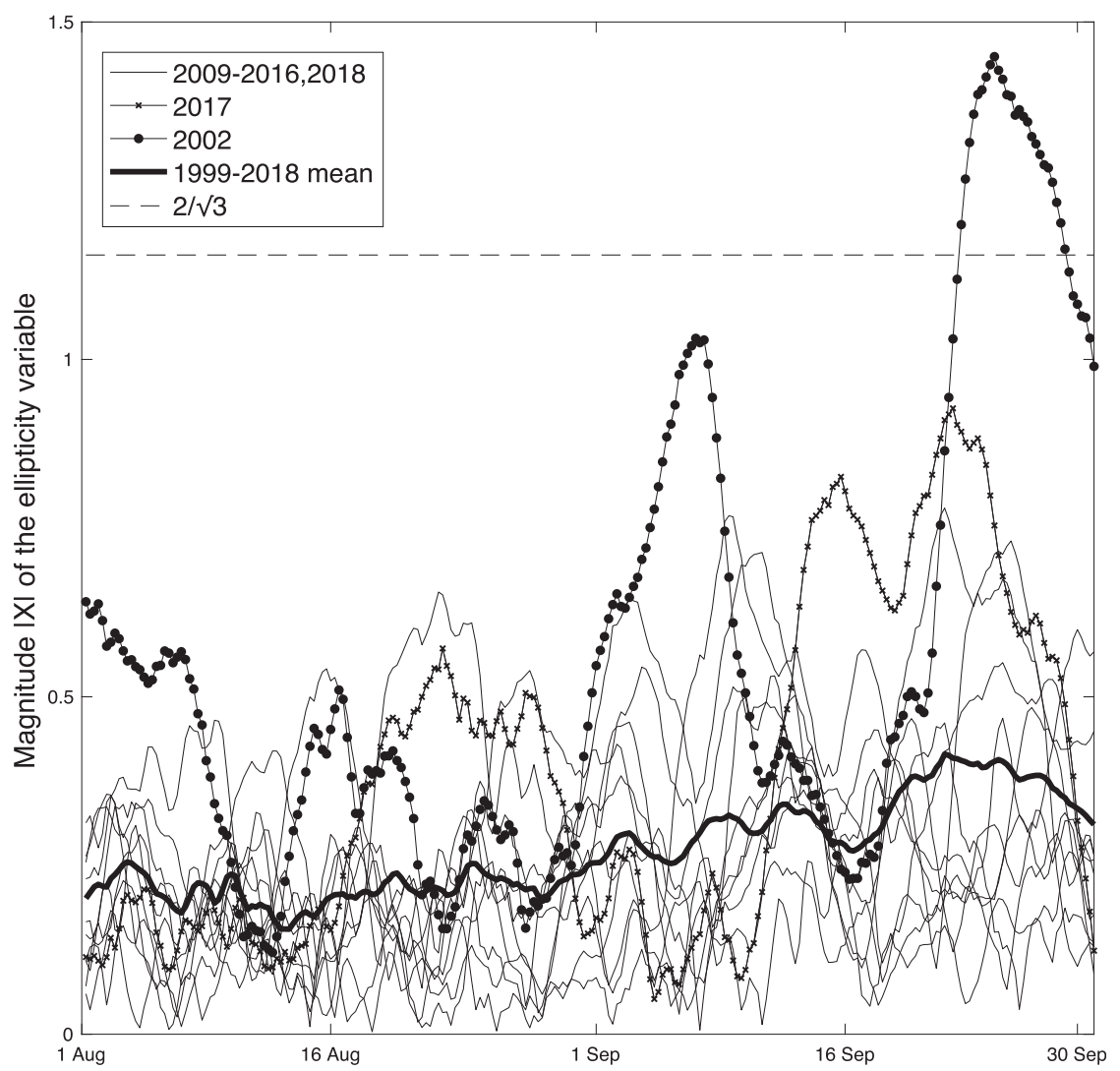

FIG. 5. Individual evolutions of the magnitude of the observed ellipticity variable $\mathbf{X}_{\mathrm{obs}}$ in 2009-18 and 2002 and its climatological behavior averaged over 1999-2018.

remains less than 0.5 , that is, much smaller than the threshold for instability. However, there is a significant upward trend throughout the entire August-September period, indicating that the vortex is becoming more disturbed as the winter progresses [cf. Fig. 11e of Waugh and Randel (1999), which shows the same climatological trend in aspect ratio during 1978-98].

Figure 6 shows the evolution in phase space of $\mathbf{X}_{\mathrm{obs}}(t)$ for each August-September period during 2009-18, and also 2002. Here it is clear that in almost every year, the vortex is describing clockwise orbits in phase space, which can be identified with those shown in the shaded regions of Fig. 2. Since the amplitude and direction of the external strain experienced by the vortex changes throughout the winter, the structure of phase space will also change (see bottom panels of Fig. 2), with closed orbits becoming more circular during periods of weak strain, and the orbits becoming more distorted and "off-center" when the strain is stronger. It is notable from Fig. 6 that

- The amplitude of the clockwise orbits vary greatly between years. Figure 6 therefore gives a simple visualization of interannual variability in the southern vortex, between winters when the vortex remains relatively undisturbed and attains particularly low temperatures, and winters such as 2017 when the vortex reaches high ellipticity.

- The large-amplitude orbits in September 2009, August 2012, September 2013, August 2017 and September 2002, which are significantly "off-center" in the phase plane, indicate the presence of a relatively strong strain field.

- The almost perfectly circular orbits in late September 2017 indicate a relatively weak strain, meaning that the region of phase space occupied by clockwise orbits is larger, and the vortex is less likely to meet the criterion (identified as the event of crossing the separatrix, following EM19) for an SSW. This gives a possible explanation of why the large-amplitude oscillations experienced by the vortex in September 2017 did not develop into an SSW.

\section{Dynamical elliptical diagnostics}

In this section a variational data assimilation technique is introduced. The aim of the technique is to use Kida's model to understand and interpret the observed 

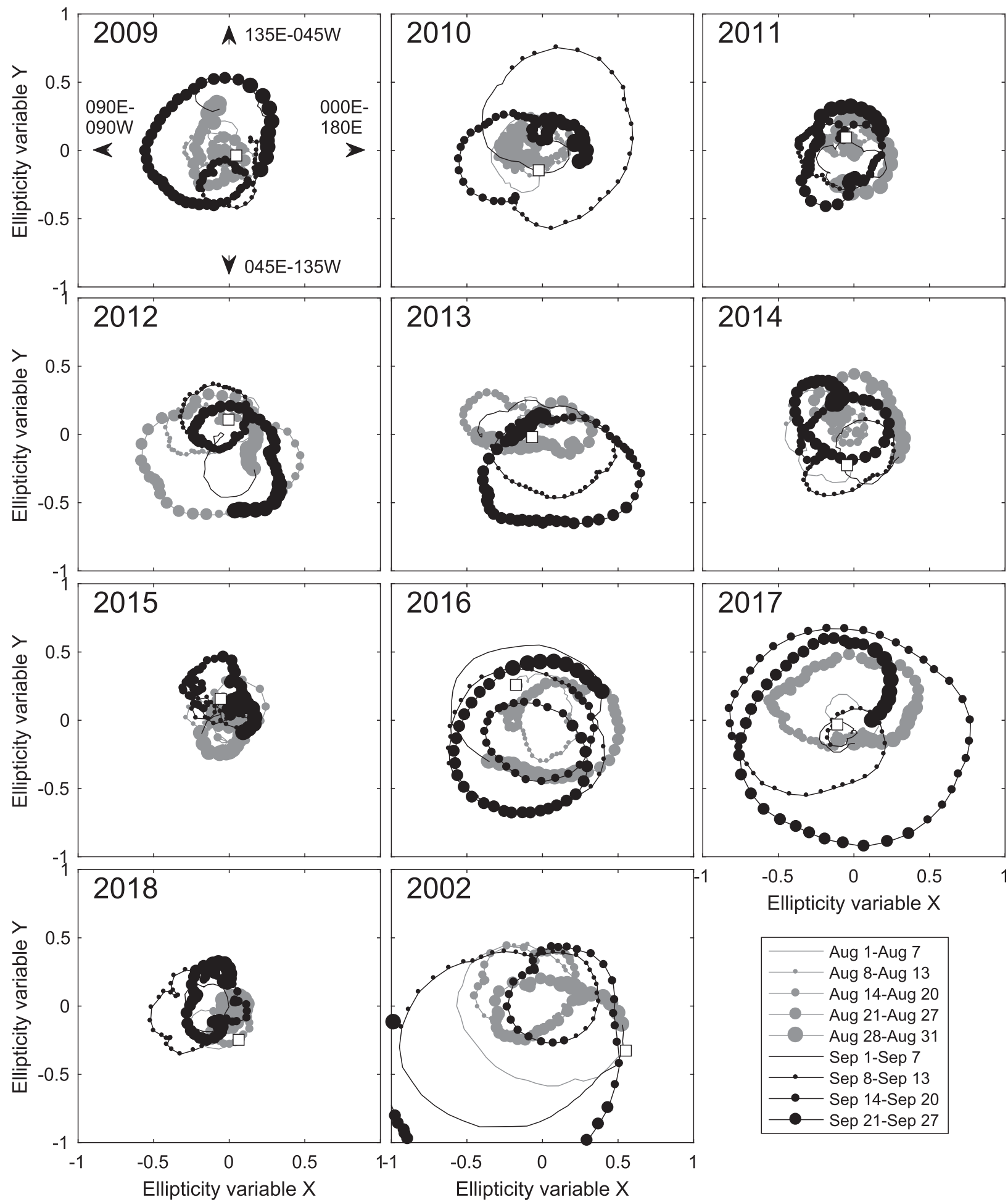

FIG. 6. The evolution of the elliptical phase-space variables $(X, Y)$ during the 10 August-September periods for the years $2009-18$ and for 2002. The white square marks the position of the ellipticity variables at the beginning of the observed time period. 
time-evolving elliptical diagnostics described above. More precisely, we will simultaneously obtain a slowly evolving strain-rotation vector $\boldsymbol{\Gamma}(t)$ that is consistent with the background forcing experienced by the vortex, and a time series of the ellipticity variable $\mathbf{X}(t)$ that is "close" to the observations $\mathbf{X}_{\text {obs }}(t)$, which together satisfy Kida's equation [(4)]. The results provide a framework to understand and interpret the observed oscillations of the vortex, as well as providing a more precise dynamical interpretation of how far the system is from a vortex-splitting SSW at any given time.

\section{The assimilation equations}

The assimilation equations are derived by first specifying a suitable cost function and then applying the standard techniques of variational calculus to determine its minimizer. The cost function, which consists of three components, is

$$
J(\boldsymbol{\Gamma}, \mathbf{X}, \boldsymbol{\mu})=\int_{0}^{T}\left[|\dot{\Gamma}|^{2}+\epsilon^{2}\left|\mathbf{X}-\mathbf{X}_{\mathrm{obs}}(t)\right|^{2}+\boldsymbol{\mu} \cdot(\dot{\mathbf{X}}-\mathbf{F})\right] d t,
$$

where $\mathbf{F}(\mathbf{X}, \boldsymbol{\Gamma}, \omega)=\nabla^{\perp} H$. The first component penalizes rapid changes in $\boldsymbol{\Gamma}(t)$, because we are interested in finding a slowly evolving $\boldsymbol{\Gamma}(t)$ that best describes the external forcing experienced by the vortex. The second component penalizes differences between the assimilated and observed ellipticity vector, and the third component introduces the Lagrange multiplier $\boldsymbol{\mu}(t)$ to ensure that the assimilated solution is a solution of Kida's equation [(4)]. The free parameter $\epsilon$ controls the extent to which $\boldsymbol{\Gamma}$ evolves slowly ( $\epsilon$ small) versus the extent to which the assimilated solution $\mathbf{X}$ remains close to the observations $\mathbf{X}_{\text {obs }}(\epsilon$ large). The time interval $T$ is the length of the observed time window (61 days; days are used as the time unit in our calculations).

The extremal functions minimizing $J$ are obtained by solving the Euler-Lagrange equations,

$$
\begin{aligned}
& \ddot{\boldsymbol{\Gamma}}=-\frac{1}{2}\left(\nabla_{\boldsymbol{\Gamma}} \mathbf{F}\right) \cdot \boldsymbol{\mu}, \\
& \dot{\boldsymbol{\mu}}=2 \epsilon^{2}\left(\mathbf{X}-\mathbf{X}_{\mathrm{obs}}\right)-(\nabla \mathbf{F}) \cdot \boldsymbol{\mu}, \\
& \dot{\mathbf{X}}=\mathbf{F} .
\end{aligned}
$$

where $\nabla_{\boldsymbol{\Gamma}}=\left(\partial_{\Gamma_{1}}, \partial_{\Gamma_{2}}, \partial_{\Gamma_{3}}\right)^{\mathrm{T}}$ is the gradient operator in the space of the vector $\boldsymbol{\Gamma}$ and $\nabla_{\boldsymbol{\Gamma}} \mathbf{F}$ denotes the $3 \times 2$ matrix with components $\partial_{\Gamma_{i}} F_{j}$. The accompanying boundary conditions are

$$
\begin{aligned}
\dot{\boldsymbol{\Gamma}}(0) & =\dot{\boldsymbol{\Gamma}}(T)=0, \\
\boldsymbol{\mu}(0) & =\boldsymbol{\mu}(T)=0 .
\end{aligned}
$$

Note that in total there are 10 boundary conditions for the coupled system of three second-order equations and four first-order equations, which is the correct number for a unique solution in a linear system.

The system (12) and (13) is solved numerically using a generalization of the shooting method, which is described in detail in the appendix. Before integrating the assimilation equations, it is necessary to choose a value of $\omega$ for the vorticity jump, for use in (4). To do this a typical value of $\omega=-6$ days $^{-1}$ is chosen based on Fig. 3 . Some sensitivity tests have been performed, using instead time-varying $\omega(t)$ calculated directly from Fig. 3, with the results being generally unchanged. All of the results below are given for $\epsilon^{2}=0.01$, which, as is shown in the sensitivity tests detailed in the appendix, gives a good compromise between the assimilated solution $\mathbf{X}(t)$ closely following the observed data $\mathbf{X}_{\mathrm{obs}}(t)$, and the inferred strain-rotation vector $\boldsymbol{\Gamma}(t)$ being slowly varying in time.

In the appendix, some explicit solutions of (12) and (13) are presented, showing the typical behavior of the first component of the ellipticity vector $\mathbf{X}(t)$ and the strain vector $\boldsymbol{\Gamma}_{1}(t)$ (see Fig. 9 there). The results show that the solutions have the desired properties of $\mathbf{X}(t)$ following $\mathbf{X}_{\mathrm{obs}}(t)$ and $\boldsymbol{\Gamma}_{1}(t)$ varying slowly. Sensitivity tests in which $\epsilon$ is varied reveal that there is only a weak sensitivity to $\epsilon$. The necessity of including a timedependent strain-rotation vector is demonstrated by freezing the strain-rotation vector at the midpoint of the assimilation time window (equivalent to applying $\epsilon=0$ in the second half of the time interval). In this case the " $\boldsymbol{\Gamma}$-frozen" solution is time periodic and, while the solution follows the observed ellipticity vector $\mathbf{X}_{\text {obs }}$ for roughly one period, it diverges rapidly from $\mathbf{X}_{\text {obs }}$ thereafter. Hence the constant- $\Gamma$ Kida equations can therefore capture the evolution over short periods, but longer periods require time-varying $\Gamma$.

\section{Results of the assimilation process}

The assimilation equations [(12) and (13)] have been solved for each August-September period from 1999 to 2018. The calculated strain-rotation vectors $\boldsymbol{\Gamma}(t)$ provide a record of the effective forcing experienced by the vortex during each winter period. All of the results shown below are for the $600-\mathrm{K}$ isentropic surface the results for which are representative of, and highly correlated with, all levels in the range 450-800 K.

Figure 7 shows the time evolution of the magnitude of the strain component $\left(\Gamma_{1}^{2}+\Gamma_{2}^{2}\right)^{1 / 2}$ and the rotation component $\Gamma_{3}$, respectively (in both cases we scale with the vorticity jump $\omega$, which is the natural nondimensionalization for Kida's equations). It is very striking that the strain component is small in magnitude and fluctuates rapidly throughout each winter with high 

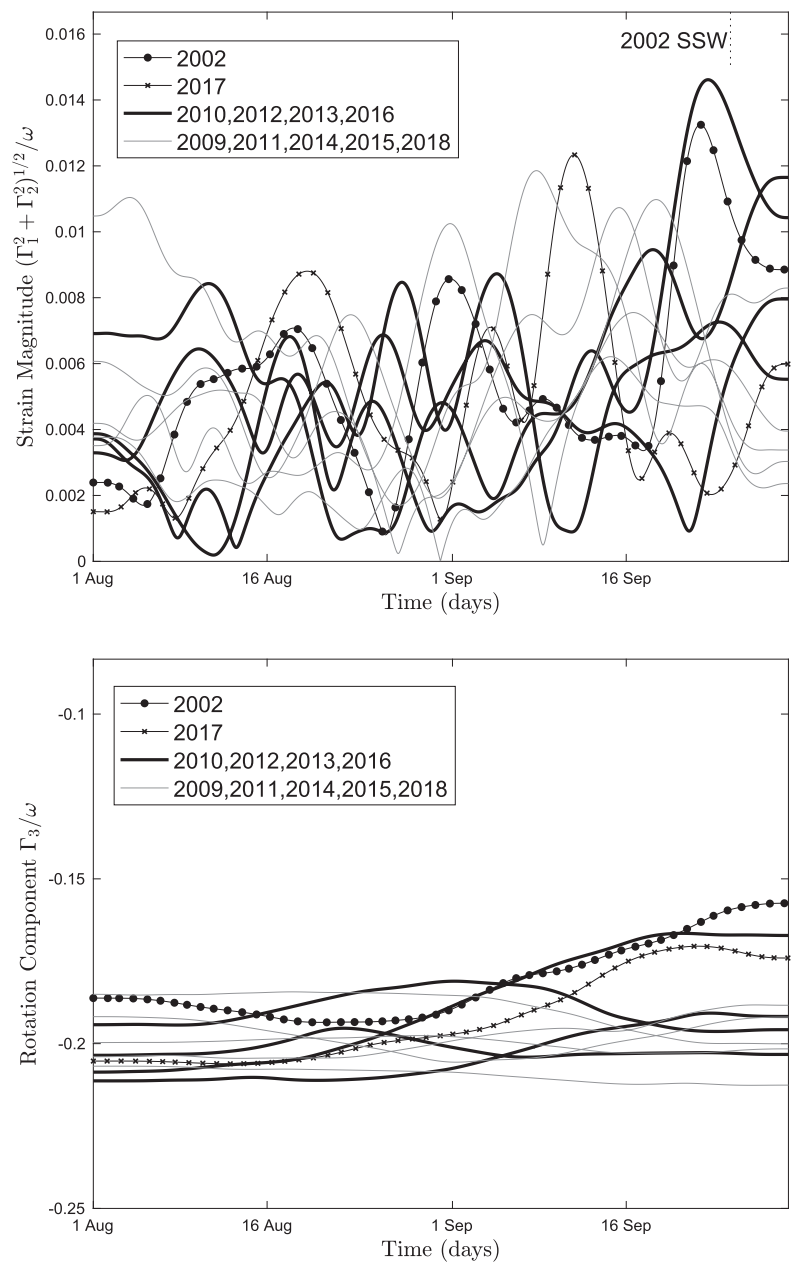

FIG. 7. (top) Time evolution of the magnitude of the strain component of the background flow $\left(\Gamma_{1}^{2}+\Gamma_{2}^{2}\right)^{1 / 2} /|\omega|$. (bottom) Time evolution of the rotation component of the background flow $\Gamma_{3} / \omega$. Both panels cover August-September for the years 2009-18 and for 2002 .

variability, whereas the rotation component of the linear flow has a large magnitude and is relatively slowly varying. The value of $\Gamma_{3}$ remains within the narrow range -0.21 to $-0.18 \omega$ during August and it has slightly increased variability -0.22 to $-0.16 \omega$ during September. These values reflect the fact that the relative vorticity outside of the vortex is positive; that is, the cyclonic polar vortex is embedded in a larger-scale anticyclonic flow. The value of $\Gamma_{3} / \omega$ found by the assimilation method is close to that used to produce "realistic" stratospheric flow profiles in previous model studies (e.g., Esler et al. 2006; EM19). The fact that the assimilation process robustly estimates $\Gamma_{3}$ at a value consistent with observations is evidence of the utility of the model. The representative value of $\Gamma_{3} / \omega=-0.2$ was used in Fig. 2, validating the relevance of the phase portraits shown.
Comparing years with a strongly elliptical vortex (2010, 2012, 2013, 2016, curves in bold; 2017 and 2002, curves with markers) with the relatively undisturbed years $(2009,2011,2014,2015,2018)$, it is clear that in general there is negligible correlation between the magnitude of the strain and the extent to which the vortex is disturbed. Admittedly, the 2002 SSW does occur following a period of high strain, and there is also a period of high strain in the next most disturbed year (2017), so extreme disturbance events may be an exception. The lack of correlation between the vortex ellipticity and the contemporaneous strain magnitude is a property of the stochastic Kida models studied in EM19. In the EM19 models, the vortex undergoes a random walk in state space in response to the random background flow, with the difference between disturbed and undisturbed winters depending on the outcome of this random walk, as opposed to being caused by individual strain events. Interestingly, there is a suggestion of a relationship between disturbed winters (particularly Septembers) and anomalously low background rotation $\Gamma_{3}$, although there are too few winters for this to be statistically significant.

Figure 8 summarizes the statistical variability of the strain components $\left(\Gamma_{1}, \Gamma_{2}\right)$. The top panel shows the mean for each August-September period (points) as well as a Gaussian fit to the distribution over the period 1999-2018 (shaded regions). The arrows at the edge of the figure give the orientation of the principal axis of the strain, relative to the lines of meridian. The most striking feature across the two panels is again the fact that there is very strong intraseasonal variability in $\left(\Gamma_{1}, \Gamma_{2}\right)$, showing that the forcing experienced by the vortex fluctuates considerably across the course of a winter. Figure 8 provides strong evidence that the southern polar vortex is much more strongly influenced by nonstationary planetary waves caused by tropospheric macroturbulence, rather than stationary planetary waves, which would have a constant orientation on Fig. 8.

Notwithstanding the last remark above, the signature of stationary forcing is nevertheless clearly apparent in Fig. 8. Comparing the mean of $\left(\Gamma_{1}, \Gamma_{2}\right)$ for each individual year, there is a clear bias toward a negative value of $\Gamma_{2}$ (and less significantly, a slightly positive value of $\left.\Gamma_{1}\right)$. The consistency of this result over the twenty year period shows there is a clear stationary wave component to the linear flow field experienced by the vortex, consisting of a strain flow roughly orientated along the $40^{\circ} \mathrm{E}^{\circ}-140^{\circ} \mathrm{W}$ meridian, of magnitude approximately $0.025|\omega|=0.15$ days $^{-1}$. The presence of this stationary strain, with fixed orientation, will act to increase the probability of Southern Hemisphere vortex splits occurring with a particular orientation (the Kida model 

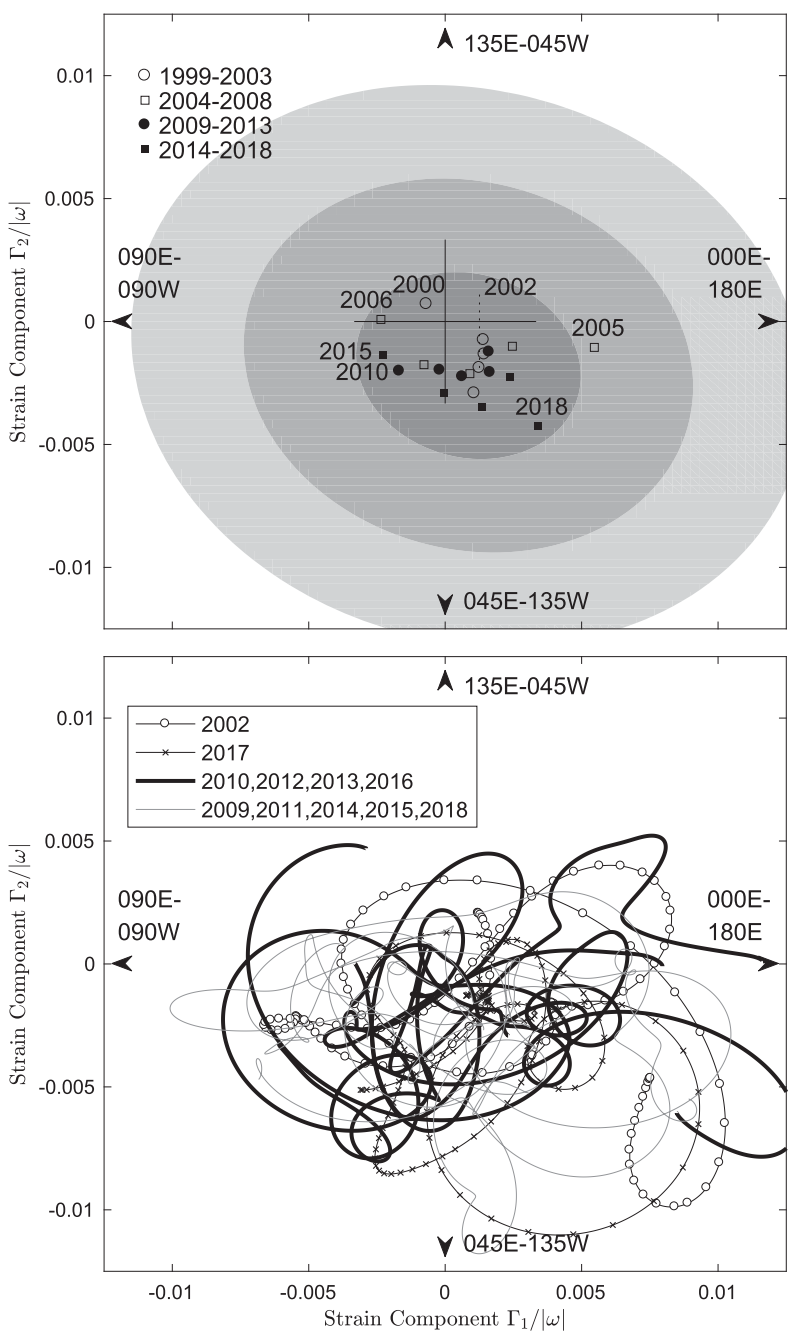

FIG. 8. (top) A Gaussian fit to the distribution of the strain component of the background flow $\left(\Gamma_{1}, \Gamma_{2}\right) /|\omega|$ during the period 1999-2018, as calculated by the assimilation process (see text) Contours are at $e^{-1 / 2}, e^{-2}$, and $e^{-9 / 2}$ times the maximum value (cf. one to three standard deviations). The means for individual years are shown as solid points (also included is 2002). (bottom) Curves showing the evolution of $\left(\Gamma_{1}, \Gamma_{2}\right) /|\omega|$ during each August-September period for years 2009-18 and for 2002 .

suggests that splits will be initiated at orientation $45^{\circ}$ clockwise of the stationary strain). However, the fact that the stationary component of the strain field is much weaker than the time varying component suggests that any bias in the direction of future vortex splits will be small, and all possible split orientations will be realized in practice.

The assimilated $\mathbf{X}(t)$ and $\boldsymbol{\Gamma}(t)$ also allow the phaseplane criterion for an SSW to be examined more precisely for each winter period. Recall that in Fig. 2 an SSW occurs in Kida's model when the phase-plane trajectory crosses the separatrix (i.e., the solid curve in the top-right panel), because the new (OSC) orbit in phase space requires the vortex to reach a much higher ellipticity, certainly exceeding Love's criterion for instability of $\lambda>3$. Crossing the separatrix would not be possible if $\boldsymbol{\Gamma}$ were time independent, because the trajectory would then be constrained, by conservation of $H$, to a closed orbit. However, because $\boldsymbol{\Gamma}$ varies in time, not only can $H$ change (e.g., due to random noise as discussed in EM19), but the position of the separatrix itself will also vary, as shown in the lower panels of Fig. 2. The value of both $H$ and its critical value $H_{c}$, which is the value of the separatrix curve, have been calculated for each winter period (not shown). The results confirm that the critical value $H=H_{c}$ is attained only in 2002, and not approached closely on other occasions, except briefly in 2009 and 2013 when the magnitude of the strain is very large, shrinking the $\mathrm{CW}$ region in phase space. Interestingly, during the period of large ellipticity in mid- to late September 2017, the Hamiltonian $H$ does not approach $H_{c}$ because the strain rate is rather low resulting in an unusually low value for $H_{c}$.

\section{Conclusions}

Here Kida's model of a two-dimensional vortex evolving in a linear background flow has been used to generalize the elliptical diagnostics of Waugh (1997) in order to allow a dynamical interpretation of the observed evolution of the Antarctic stratospheric polar vortex. A Hamiltonian reformulation of Kida's equations allows for a phasespace interpretation of the periodic orbits performed by the vortex, and in particular highlights the amplitude bifurcation, which has been previously associated with SSWs (Matthewman et al. 2009). Analysis of the vortex behavior during 1999-2018 showed that in every year the vortex near continuously rotates clockwise or nutates, consistent with these periodic orbits of the Kida vortex.

Variational data assimilation was then used to infer the linear background flow experienced by the Antarctic vortex as it evolved over each August-September period. The main findings are as follows:

- The rotational component of the background flow is slowly evolving and relatively large in magnitude, undergoes only moderate year-to-year variability, and is consistent with the cyclonic polar vortex being embedded in an anticyclonic background flow (cf. Esler and Scott 2005; Esler et al. 2006).

- The strain component of the background flow evolves relatively rapidly (on a characteristic time scale of a week or so), is relatively weak, and is dominated by its unsteady component. 
- A steady component to the strain flow is clearly detectable and persistent between years, and is aligned with the $40^{\circ} \mathrm{E}-140^{\circ} \mathrm{W}$ meridian and has a relatively small magnitude $\left(\approx 0.15\right.$ days $\left.^{-1}\right)$. This is the signature of the effect of stationary planetary waves on the evolving vortex. [See the discussion in, e.g., Esler and Scott (2005) or EM19 for an explanation of how planetary-scale topography can be identified with an external strain flow at the level of the stratospheric vortex.] It is interesting to note that the streamfunction associated with this strain flow has a tendency to enhance anticyclonic motion in the region of the "Australian High" consistent with the anticyclonic flow often observed there [see, in particular, Harvey et al. (2002)], which suggests a link between the obtained climatological strain flow and the development of pairs of eastward-traveling anticyclones that is well documented in literature (Harvey et al. 2002; Lahoz et al. 1996).

The fact that the time-varying component of the strain flow is much larger than the stationary component implies that it is the dynamically active troposphere that ultimately controls the frequency and nature of Antarctic SSWs, as opposed to stationary planetary waves due to land-sea contrast, etc., which are much more important in the Arctic. The archetypal "intermediate complexity" model of an Antarctic SSW is therefore, arguably, the GCM study of Kushner and Polvani (2005), in which an SSW occurs spontaneously in a long simulation in the absence of planetary wave forcing from the lower boundary. For further evidence of the relevance of Kida's model, or similar simple reduced models of SSWs, to the Antarctic stratosphere, it will be necessary to reexamine intermediate complexity models such as Kushner and Polvani (2005).

The key question will be whether a free-running version of Kida's model such as those studied in EM19, forced by a statistical model of the background flow trained on the GCM data, is capable of meaningfully predicting the statistics of vortex ellipticity, and the resulting probabilities of vortex splits. The influence of stationary waves can also be considered, by gradual addition of topographic forcing; compare Taguchi et al. (2001) and Sheshadri et al. (2015). Such a stochastic model, once established, can be used to make predictions about how key climatological variables such as SSW frequency vary under changes to the model setup and parameters. It also provides a simple pathway to understanding why the changes occur, as changes to the model climate can be easily linked to changes in the external background flow experienced by the vortex. From there, a deeper understanding of how SSW behavior will change under a changing climate will be attainable. More broadly, the methodology we have introduced will be useful in the study of vortices elsewhere on Earth and in the solar system more widely (cf. Polvani et al. 1990; Mitchell et al. 2015).

Acknowledgments. JGE acknowledges support from the U.K. Natural Environment Research Council Grant NE/S00985X/1 and MM the support of the EPSRC Studentship Award 1777889.

\section{APPENDIX}

\section{Solution of the Assimilation Equations}

\section{a. The numerical algorithm}

Here, the algorithm used to solve the Euler-Lagrange equation [(12)] with boundary conditions (13) is described. It is nontrivial to find a solution to (12) satisfying (13), because a naive attempt to integrate (12) over the interval $[0, T]$ results in a floating-point overflow, due to the solution exploding exponentially with a growth rate of a few hours. In other words, to solve (12) and (13) by forward integration requires an extremely precise guess for the unknown initial conditions for $\boldsymbol{\Gamma}(0)$ and $\mathbf{X}(0)$, which must be obtained iteratively.

Our algorithm generalizes the well-known shooting method, used for solving boundary problems for ordinary differential equations, as follows. First, the equations are rewritten as a first-order system by introducing notation $\boldsymbol{\zeta}=\dot{\boldsymbol{\Gamma}}$ and $\mathbf{Z}=(\boldsymbol{\Gamma}, \boldsymbol{\zeta}, \boldsymbol{\mu}, \mathbf{X})^{\mathrm{T}}$ to give

$$
\dot{\mathbf{Z}}=\mathbf{G}(\mathbf{Z}, t),
$$

where

$$
\mathbf{G}(\mathbf{Z}, t)=\left(\begin{array}{c}
\boldsymbol{\zeta} \\
-\frac{1}{2}\left(\nabla_{\Gamma} \mathbf{F}\right) \cdot \boldsymbol{\mu} \\
2 \epsilon^{2}\left[\mathbf{X}-\mathbf{X}_{\mathrm{obs}}(t)\right]-(\nabla \mathbf{F}) \cdot \boldsymbol{\mu} \\
\mathbf{F}
\end{array}\right) .
$$

The corresponding boundary conditions are now

$$
\begin{aligned}
\zeta(0) & =\boldsymbol{\zeta}(T)=0, \\
\boldsymbol{\mu}(0) & =\boldsymbol{\mu}(T)=0 .
\end{aligned}
$$

Next, the time domain is divided into $N$ equal intervals $\left[t_{k}, t_{k+1}\right]$ for $k=0, \ldots, N-1$, where $t_{k}=k T / N$. The solution to (A1) on the interval $\left[t_{k}, t_{k+1}\right]$ will be denoted $\mathbf{Z}_{k}(t)$. A vector $\mathbf{S}$ of initial guesses, for the solution at the beginning of each subinterval, is constructed by concatenation 


$$
\mathbf{S}=\left[\boldsymbol{\Gamma}_{0}(0), \mathbf{X}_{0}(0), \mathbf{Z}_{1}\left(t_{1}\right), \ldots, \mathbf{Z}_{N-1}\left(t_{N-1}\right)\right]^{\mathrm{T}} .
$$

The vector $\mathbf{S}$ has $10 N-5$ components, since $\zeta_{0}(0)=0$ and $\boldsymbol{\mu}_{0}(0)=0$ are specified.

A standard ODE solver (we use MATLAB's ode45 with a small absolute error tolerance of $10^{-8}$ ) is then used to solve for $\mathbf{Z}_{k}(t)$ on each interval $\left[t_{k}, t_{k+1}\right]$ using the initial conditions contained in $\mathbf{S}$. The resulting solutions are of course discontinuous at every end-point $t_{k}$, and in fact the "solution mismatch" $\mathbf{W}$ can be stored in a single vector

$$
\mathbf{W}=\left(\begin{array}{c}
\mathbf{Z}_{1}\left(t_{1}\right)-\mathbf{Z}_{0}\left(t_{1}\right) \\
\vdots \\
\mathbf{Z}_{N-1}\left(t_{N-1}\right)-\mathbf{Z}_{N-2}\left(t_{N-1}\right) \\
\boldsymbol{\zeta}_{N-1}\left(t_{N}\right) \\
\boldsymbol{\mu}_{N-1}\left(t_{N}\right)
\end{array}\right)
$$

Notice that $\mathbf{W}$ also has $10 N-5$ components, with the final 5 being the boundary values in (13) at $t=t_{N}=T$.

Finding a solution to the original problem (12) and (13) is now equivalent to finding a particular set of initial conditions $\mathbf{S}=\mathbf{S}_{*}$ for which the solution mismatch $\mathbf{W}$ is zero, that is, solving the nonlinear system

$$
\mathbf{W}(\mathbf{S})=0 .
$$

The problem (A6) can be solved by implementing a standard root finding algorithm in multiple dimensions. The problem is standard except that each evaluation of the function $\mathbf{W}$ requires a new solution for $\mathbf{Z}_{k}(t)(k=0, \ldots, N-$ 1). Here, the trust-region dogleg algorithm (inbuilt solver fsolve in MATLAB) is used to find a solution to accuracy $|\mathbf{W}|<\delta$, which is chosen to be $10^{-8}$. The initial values used for the first guess of $\mathbf{S}$ are $\Gamma_{1}\left(t_{k}\right)=\Gamma_{2}\left(t_{k}\right)=0, \Gamma_{3}\left(t_{k}\right)=$ $\Omega=-\omega / 5, \boldsymbol{\zeta}\left(t_{k}\right)=0, \boldsymbol{\mu}\left(t_{k}\right)=0$, and $\mathbf{X}\left(t_{k}\right)=\mathbf{X}_{\mathrm{obs}}\left(t_{k}\right)$.

The above algorithm will converge rather slowly unless the following speedup is applied. Notice that the Jacobian matrix $\mathbf{J}$ of (A6), which has components $J_{i j}=$ $\partial W_{i} / \partial S_{j}$, has a highly simplified structure, with nonzero entries only on two "block" diagonals. This is because the solution mismatches $\mathbf{Z}_{k}\left(t_{k}\right)-\mathbf{Z}_{k-1}\left(t_{k}\right)$ in $\mathbf{W}$ depend only on the initial conditions $\mathbf{Z}_{k-1}\left(t_{k-1}\right)$ and $\mathbf{Z}_{k}\left(t_{k}\right)$ in $\mathbf{S}$. A standard implementation of most nonlinear root finding methods requires repeated numerical evaluation of the Jacobian, which will necessarily involve enormous redundancy if the "zero" terms in $\mathbf{J}$ are calculated numerically. This redundancy can be avoided by supplying a routine to calculate the simplified Jacobian explicitly.

\section{b. Solution behavior and parameter sensitivities}

Figure A1 shows some examples of the solutions of (12) and (13) obtained using the above algorithm. All
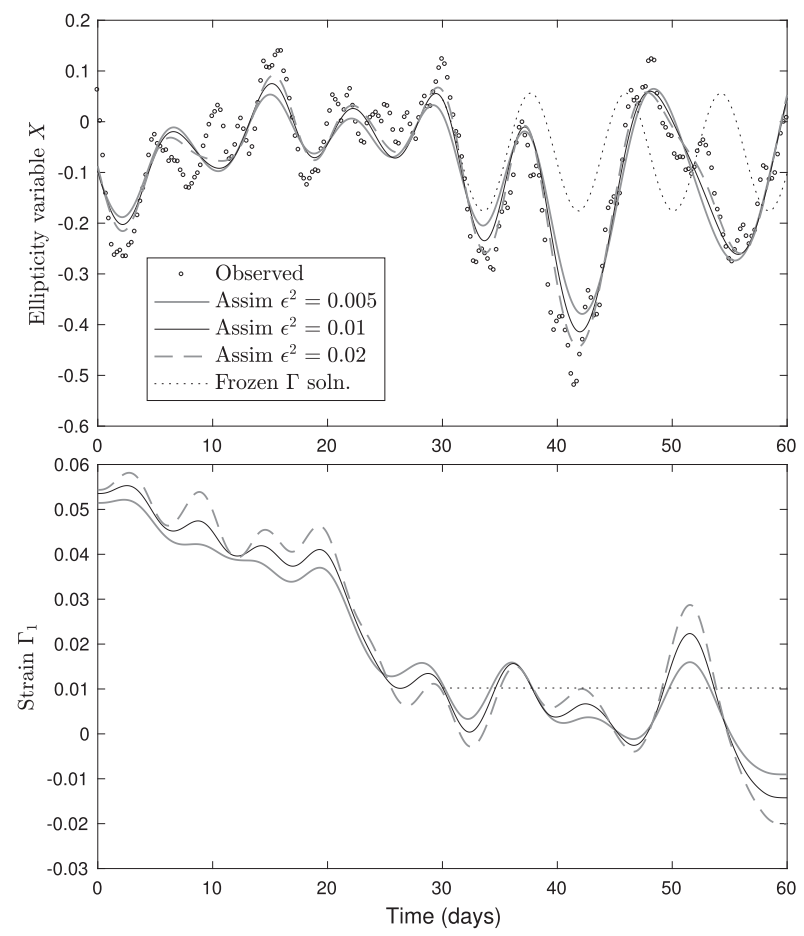

FIG. A1. (top) Ellipticity variable $\mathbf{X}(t)$ for August-September 2018, in the observations (points) and the assimilated solutions (curves), showing the effect of the parameter $\epsilon$. (bottom) The first component $\Gamma_{1}$ of the strain-rotation vector for the same solutions. In each panel the dotted curve shows the solution of Kida's equations when the strain-rotation vector is frozen at $t=30$ days (in the $\epsilon^{2}=0.01$ solution).

results shown are obtained with $N=60$ subintervals, with some solutions checked against solutions with $N=30$ to verify the robustness of the algorithm, with agreement found within the chosen tolerance $\delta$. The solution components shown are $\mathbf{X}(t)$ and $\Gamma_{1}(t)$ for August-September 2018. The observed values $\mathbf{X}_{\mathrm{obs}}(t)$ for the ellipticity variable are plotted as solid circles, and the assimilated solutions are curves.

Specifically, Fig. A1 shows the effect of varying $\epsilon$, the free parameter in the cost function. The contrasting limits $\epsilon \rightarrow 0$ and $\epsilon \rightarrow \infty$ can be explained as follows. Solution with $\epsilon \rightarrow 0$ have constant strain-rotation vector $\boldsymbol{\Gamma}$, corresponding to the limit in which the background forcing is constant, and the assimilated solution best fitting the observations is periodic in time. Such a solution is generally a poor fit. The limit $\epsilon \rightarrow \infty$ corresponds to the case where measurements and model assumptions are perfect and the assimilated solution interpolates the observations precisely. The problem in this case is that the strain-rotation vector $\boldsymbol{\Gamma}$ now varies extremely rapidly in time and defies interpretation. The solutions shown therefore have intermediate values of $\epsilon\left(\epsilon^{2}=\right.$ $0.005,0.01$, and 0.02 ), and result in assimilated solutions 
that both fit the observations reasonably closely, and have smoothly varying $\Gamma(t)$. The effect of increasing $\epsilon$ is now clear. At higher $\epsilon$ the assimilated solutions fit the observed data more closely, but at the expense of $\boldsymbol{\Gamma}$ varying more rapidly in time.

Also shown in Fig. A1 is a solution to Kida's equations in which the strain-rotation vector $\boldsymbol{\Gamma}$ is frozen halfway through the solution at $t=30$ days. This solution is included in order to demonstrate that Kida's equations with constant $\boldsymbol{\Gamma}$ do a good job of qualitatively capturing the CW cycles performed by the vortex. The constant $\boldsymbol{\Gamma}$ solution follows the observations reasonably closely for one cycle, and then diverges as it remains on a fixed periodic orbit, while the observed solution experiences changes to the background forcing and begins to undergo orbits with larger amplitude and longer periods.

\section{REFERENCES}

Black, R. X., and B. A. McDaniel, 2007: Interannual variability in the Southern Hemisphere circulation organized by stratospheric final warming events. J. Atmos. Sci., 64, 2968-2974, https://doi.org/10.1175/JAS3979.1.

Dee, D. P., and Coauthors, 2011: The ERA-Interim reanalysis: Configuration and performance of the data assimilation system. Quart. J. Roy. Meteor. Soc., 137, 553-597, https://doi.org/ 10.1002/qj.828.

Dritschel, D. G., 1990: The stability of elliptical vortices in an external straining flow. J. Fluid Mech., 210, 223-261, https:// doi.org/10.1017/S0022112090001276.

Esler, J. G., and R. K. Scott, 2005: Excitation of transient Rossby waves on the stratospheric polar vortex and the barotropic sudden warming. J. Atmos. Sci., 62, 3661-3682, https://doi.org/ 10.1175/JAS3557.1.

_ and M. Mester, 2019: Noise-induced vortex splitting stratospheric sudden warmings. Quart. J. Roy. Meteor. Soc., 145, 476-494, https://doi.org/10.1002/qj.3443.

- L. M. Polvani, and R. K. Scott, 2006: The Antarctic stratospheric sudden warming of 2002: A self-tuned resonance? Geophys. Res. Lett., 33, L12804, https://doi.org/10.1029/ 2006GL026034.

Hannachi, A., D. M. Mitchell, L. Gray, and A. J. Charlton-Perez, 2011: On the use of geometric moments to examine the continuum of sudden stratospheric warmings. J. Atmos. Sci., 68 , 657-674, https://doi.org/10.1175/2010JAS3585.1.

Harvey, V. L., R. B. Pierce, and M. H. Hitchman, 2002: A climatology of stratospheric polar vortices and anticyclones. J. Geophys. Res., 107, 4442, https://doi.org/10.1029/ 2001JD001471.

Kida, S., 1981: Motion of an elliptic vortex in a uniform shear flow. J. Phys. Soc. Japan, 50, 3517-3520, https://doi.org/10.1143/ JPSJ.50.3517.

Kushner, P. J., and L. M. Polvani, 2005: A very large, spontaneous stratospheric sudden warming in a simple AGCM: A prototype for the Southern Hemisphere warming of 2002 ? J. Atmos. Sci., 62, 890-897, https://doi.org/10.1175/JAS-3314.1.

Lahoz, W. A., and Coauthors, 1996: Vortex dynamics and the evolution of water vapour in the stratosphere of the Southern Hemisphere. Quart. J. Roy. Meteor. Soc., 122, 423-450, https:// doi.org/10.1002/qj.49712253007.

Love, A. E. H., 1893: On the stability of certain vortex motion. Proc. Soc. London, 23, 18-42.

Matthewman, N. J., and J. G. Esler, 2011: Stratospheric sudden warmings as self-tuning resonances. Part I: Vortex splitting events. J. Atmos. Sci., 68, 2481-2504, https://doi.org/10.1175/JAS-D-11-07.1.

,$- \ldots$, A. J. Charlton-Perez, and L. M. Polvani, 2009: A new look at stratospheric sudden warmings. Part III. Polar vortex evolution and vertical structure. J. Climate, 22, 1566-1585, https://doi.org/10.1175/2008JCLI2365.1.

Melander, M. V., N. J. Zabusky, and A. S. Styczek, 1986: A moment model for vortex interactions of the two-dimensional Euler equations. Part 1. Computational validation of a Hamiltonian elliptical representation. J. Fluid Mech., 167, 95-115, https://doi.org/10.1017/S0022112086002744.

Mitchell, D. M., A. J. Charlton-Perez, and L. J. Gray, 2011: Characterizing the variability and extremes of the stratospheric polar vortices using 2D moment analysis. J. Atmos. Sci., 68, 1194-1213, https://doi.org/10.1175/2010JAS3555.1.

_ , and Coauthors, 2012: The nature of Arctic polar vortices in chemistry-climate models. Quart. J. Roy. Meteor. Soc., 138, 1681-1691, https://doi.org/10.1002/qj.1909.

_ L. Montabone, S. Thomson, and P. Read, 2015: Polar vortices on Earth and Mars: A comparative study of the climatology and variability from reanalyses. Quart. J. Roy. Meteor. Soc., 141, 550-562, https://doi.org/10.1002/qj.2376.

Polvani, L. M., J. Wisdom, E. DeJong, and A. P. Ingersoll, 1990: Simple dynamical models of Neptune's great dark spot. Science, 249, 1393-1398, https://doi.org/10.1126/science.249.4975.1393.

Scaife, A. A., D. R. Jackson, R. Swinbank, N. Butchart, H. E. Thornton, M. Keil, and L. Henderson, 2005: Stratospheric vacillations and the major warming over Antarctica in 2002. J. Atmos. Sci., 62, 629-639, https://doi.org/10.1175/JAS-3334.1.

Scinocca, J. F., and P. H. Haynes, 1998: Dynamical forcing of stratospheric planetary waves by tropospheric baroclinic eddies. J. Atmos. Sci., 55, 2361-2392, https://doi.org/10.1175/15200469(1998)055<2361:DFOSPW >2.0.CO;2.

Sheshadri, A., R. A. Plumb, and E. P. Gerber, 2015: Seasonal variability of the polar stratospheric vortex in an idealized AGCM with varying tropospheric wave forcing. J. Atmos. Sci. 72, 2248-2266, https://doi.org/10.1175/JAS-D-14-0191.1.

Taguchi, M., T. Yamaga, and S. Yoden, 2001: Internal variability of the troposphere-stratosphere coupled system simulated in a simple global circulation model. J. Atmos. Sci., 58, 3184-3203, https:// doi.org/10.1175/1520-0469(2001)058<3184:IVOTTS >2.0.CO;2.

Waugh, D. W., 1997: Elliptical diagnostics of stratospheric polar vortices. Quart. J. Roy. Meteor. Soc., 123, 1725-1748, https:// doi.org/10.1002/qj.49712354213.

, and W. J. Randel, 1999: Climatology of Arctic and Antarctic polar vortices using elliptic diagnostics. J. Atmos. Sci., 56, 1594-1613, https://doi.org/10.1175/1520-0469(1999)056<1594: COAAAP $>2.0 . \mathrm{CO} ; 2$. 